\title{
Effectiveness of an Unexpected Disturbance Program in the Early Stage of Rehabilitation in Athletes With Unilateral Knee Ligament Injury
}

\author{
Joerg Teichmann, Rachel Tan, Kim Hébert-Losier, Yeo Wee Kian, Shabana Jalal Din, \\ Ananthi Subramaniam, Dietmar Schmidtbleicher, and C. Martyn Beaven
}

\begin{abstract}
Context: Sensorimotor, proprioceptive, and neuromuscular programs are critical for the successful rehabilitation of injured athletes, and these decrease reinjury rates. Objective: To investigate the effects of an unexpected disturbance program (UDP) on balance and unilateral strength metrics in athletes with unilateral knee ligament injury. Design: A 3-week parallel-group experimental design consisting of 9 rehabilitation sessions. Setting: National Sports Institute. Participants: Twenty-one national-level athletes (age 21.4 [4.4] y, body mass 63.9 [10.8] kg, height 169.0 [10.2] cm) who had sustained a unilateral knee ligament injury. Intervention: An UDP program designed to evoke rapid sensorimotor responses was compared with traditional training and a nonexercise control group. Main Outcome Measures: Unilateral total, anteroposterior, and mediolateral sway with eyes open and closed and unilateral isometric strength. Results: Traditional exercises tended to outperform the UDP when unilateral balance testing was performed with eyes open; however, balance improvement following UDP tended to be greater in the eyes-closed condition. Significant strength gains in both the injured and uninjured legs were only observed following the UDP. This increase in unilateral isometric strength was 23.4 and $35.1 \mathrm{~kg}$ greater than the strength improvements seen in the traditional rehabilitation and control groups $(P<.05)$. Conclusions: UDP could improve neural aspects of rehabilitation to improve rehabilitation outcomes by improving strength, sensorimotor function, and proprioception. Given the complementary adaptations, an UDP could provide an effective adjunct to traditional rehabilitation protocols and improve return-to-play outcomes.
\end{abstract}

Keywords: perturbation, return to play, reinjury, sensorimotor, proprioception

In activities of daily life, we are constantly required to respond to external perturbations to maintain balance, such as when walking on wet or uneven surfaces. In sport, additional rapid unplanned changes in direction and dynamic interactions with a ball or opponent place extreme demands on sensorimotor function. An unexpected disturbance program (UDP) has previously been reported to improve outcomes in both uninjured ${ }^{1}$ and injured athletes. ${ }^{2}$ In both instances, a positive impact on explosive actions was apparent. In the injured population, perceived confidence improved (ie, kinesiophobia decreased).

Typically, the rehabilitation follows a process of 5 general phases, namely restoring the integrity, regaining the mobility, acquiring muscular strength and reflex actions, integrating sport specific movements, and finally return to play incorporating prevention exercises. ${ }^{3}$ However, the effectiveness of current rehabilitation practices and return-to-play assessments can be questioned as studies report reinjury rates as high as $49 \%{ }^{4}$ Only 55\% of 94 patients reported to successfully return to preinjury levels of sports participation 1 year postsurgery, ${ }^{5}$ and the risk of sustaining an identical knee joint injury nearly tripled in football players in the

Teichmann, Tan, Jalal Din, and Subramaniam are with the Sports Medicine Division, National Sports Institute, Kuala Lumpur, Malaysia. Hébert-Losier and Beaven are with the Division of Health, Engineering, Computing, \& Science, School of Health, Sport and Human Performance, The University of Waikato, Tauranga, New Zealand. Kian is with the Division of Research and Innovation, National Sports Institute, Kuala Lumpur, Malaysia. Schmidtbleicher is with the Department of Sport Science, Johann Wolfgang Goethe University, Frankfurt, Germany. Teichmann (jorg@rehamedtherapy.com.my) is corresponding author. season following injury. ${ }^{6}$ Conventional rehabilitation methodology focuses on muscular strength training using trampolines, balance boards, and movements performed on a compliant surface, and training movements at a relatively slow frequency to the sensorimotor system ( $>350 \mathrm{~ms})$. Given that empirical observations suggest that ruptures of ligaments in the ankle and knee joints occur in a time span of $<200 \mathrm{~ms},{ }^{7}$ the sensorimotor system may require specific training to protect against subsequent injury.

Unexpected disturbances (such as a random physical push from an unknown direction before landing from a drop jump) evoke these faster responses in the sensorimotor system. ${ }^{1}$ Unexpected disturbances likely replicate the condition athletes find themselves during training and competition. As such, these disturbances add a level of ecologically validity and a functional aspect to the sport-specific exercise element of the existing rehabilitation programs. ${ }^{1}$ To ensure an athlete is physically competent to progress to the sports-specific phase of rehabilitation (phase 4), we suggest that it is necessary to assess the athlete in movements that incorporate rapid sensorimotor perturbations during phase 3 of rehabilitation, where the focus is on muscle strengthening and restoring reflex actions.

Given that sensorimotor, proprioceptive, and neuromuscular programs are critical to successful rehabilitation, the current investigation compared 2 programs with and without unexpected disturbance during a 3-week period constituting phase 3 of the rehabilitation process. We hypothesized that the unexpected disturbance group would improve balance and unilateral strength to a greater extent than the traditionally rehabilitated athletes by imposing greater adaptive stimuli to the sensorimotor, vestibular, and proprioceptive systems. 


\section{Methods}

\section{Participants}

A total of 31 participants (20 males and 11 females) were recruited to participate in a parallel-group interventional study. Twenty-one participants met the inclusion criteria as national-level athletes (mean [SD]; age 21.4 [4.4] y, body mass 63.9 [10.8] kg, height $169.0[10.2] \mathrm{cm}$ ) of different sporting backgrounds who had sustained a unilateral knee ligament injury (18 anterior crutiate ligament [ACL] reconstructions, 2 medial collateral ligament tears, and 1 posterior cruciate ligament tear) and were currently in phase 3 of a rehabilitation program. For the purpose of this study, the participants were deemed fit for phase 3 if they had the ability to jog pain free, single-leg leg press more than $100 \%$ of body mass and could maintain balance for more than 20 seconds on their injured leg. Of these 21 participants, 19 had undergone arthroscopic surgery and 2 had undertaken nonsurgical conservative treatment.

\section{Design}

The injured participants were randomly assigned to one of the 2 groups using simple randomization: the experimental group that performed an UDP $(n=12 ; 10$ males and 2 females $)$ and a traditional training group $(n=9 ; 5$ males and 4 females). The remaining 10 participants were uninjured controls (age 24.4 [1.2] y, body mass 66.0 [13.2] kg, height 168.3 [9.0] cm) who performed no exercise intervention. This study was performed in accordance with the International Journal of Sports Medicine ethical standards document and in the spirit of the Helsinki Declaration. All the subjects were informed of the risks and procedures involved, and gave their written consent prior to participation. The National Sports Institute research and ethics committee reviewed and approved the protocol.

\section{Procedures}

Rehabilitation Program. Following the successful completion of phase 2 of the rehabilitation process (as outlined above), the athletes began a 3 -week exercise rehabilitation program. All the exercises were conducted and overseen by the same therapist to standardize the training intervention, and exercises were performed only on the injured leg. Each session lasted 45 minutes and the sessions were performed 3 times per week. Thus, a total of 9 sessions were completed. Each session consisted of 5 rehabilitative exercises that were performed either with or without the incorporation of an unexpected disturbance. The control group did not partake in any form of exercise in the 3 weeks between pretest and posttest.

Robo-Pong (Table Tennis). Participants stood on their injured leg in front of the Newgy Robo-Pong ${ }^{\circledR}$ (Hendersonville, TN) Table Tennis Robot machine and attempted to hit a table tennis ball toward a target. Five sets of 20 table tennis balls were used for both groups. For the UDP group, the machine head was set to swivel $180^{\circ}$ and expelled the ball in a random direction. For the traditional training group, the device head remained static and the direction of the ball was consistent.

Single-Leg Balancing. Participants stood upright on their injured leg (arms akimbo with the opposite leg flexed $90^{\circ}$ at the hip and the knee) for 30 seconds. This exercise was repeated 5 times with 30 -second rest in between each effort. Perturbation for the UDP group was applied from behind, with the therapist pushing the participant at the thoracolumbar (T1-L3) region at random time intervals throughout the 30 seconds. No perturbation was applied in the traditional training group.

Single-Leg Sit to Stand. Participants started by sitting on the edge of a box with their injured leg on the floor and arms akimbo. The height of the box was altered such that the injured knee was flexed at $70^{\circ}$ when seated. Participants were then asked to stand upright on the injured leg before returning to a seated position onto the box. This exercise was performed for 5 sets of 8 repetitions with 1 minute of rest between sets. Perturbation was applied from behind, with the therapist pushing the participant at the cervicothoracic region (C7-T12) at random time intervals throughout the sets. No perturbation was applied in the traditional training group.

Jogging. Participants jogged back and forth along a 20-m line in a gymnasium for 2 minutes, 5 times, with 2 minutes of rest between jogging efforts. A bell was used to give directional cues: 1 ring for forward running, 2 rings for backward running, and 3 rings for side step. For the UDP group, directional cues were given in a random order throughout each 2-minute jogging effort. No directional cues were given for the traditional training group, with the participant jogging in a line for the 2 minutes.

Stochastic Resonance Training. Participants in the UDP group were instructed to stand upright (arms akimbo and looking forward) with their injured leg on the stochastic resonance training (SRT) machine (Zeptoring Deutschland GmbH, Berlin, Germany) that produces a nonsinusoidal, random vibratory pattern. The SRT machine was set to Trim: 4, duration: 30 seconds, rest: 30 seconds, noise: 1 , and 5 repetitions were performed. Participants were instructed to volley a soccer ball back to the thrower with the uninjured leg for 30 seconds. For the traditional training group, participants stood on the SRT machine and volleyed the soccer ball back to the thrower, but the SRT machine was not turned on.

\section{Assessments}

Before and after the 3-week intervention program, the 21 athletes and 10 control participants were assessed on single-leg balance and unilateral isometric leg strength as described below.

Single-Leg Balance Test. Participants stood shod on 1 leg with an upright posture on an " $\mathrm{X}$ " marked in the middle of a force platform (Kistler-9286BA; Kistler, Winterthur, Switzerland) for 20 seconds while looking straight ahead. The nonsupporting leg was flexed at $90^{\circ}$ at the hip and knee with arms akimbo. For open eyes unilateral stance, the pretest and posttest were conducted with 3 repeated trials each with the best score taken for our results. The total sway distance, anteroposterior (AP) sway distance, and mediolateral (ML) sway distance were obtained (in millimeters) using the Kistler Measurement, Analysis and Reporting Software (MARS, version 2.1.0.8; Ljubljana, Slovenia). For closed eyes unilateral stance, the pretest and posttest were conducted with only one trial as participants found it difficult to maintain the stance for 20 seconds. All participants were instructed to refrain from intensive exercises for 24 hours prior to the test. These tests were repeated for each leg. If the participant lost balance, moved their arms, or moved their flexed leg from the start position, the trial was repeated after a 1-minute rest.

Isometric Single-Leg Press Test. The isometric unilateral horizontal leg press test was performed using the Proxomed ${ }^{\circledR}$ Compass 600 leg press machine (Medizintechnik GmbH, Alzenau, Germany). Participants were positioned on the machine with their knee flexed at $90^{\circ}$ and foot on the footplate, with the opposite leg not in contact 
with any object. Participants were then instructed to push against the footplate with maximal effort for 5 seconds. This leg press exercise was performed 3 times with 2-minute rest between each trial. The average maximum force (in kilograms) of the 3 trials was recorded for analysis. The same test was repeated with the opposite leg.

\section{Statistical Analyses}

The dependent variables were log transformed for the purpose of analysis. Back transformation provided estimates of mean effects of the 3-week intervention (ie, premeasures vs postmeasures) as percentages and errors as coefficients of variation. Analyses of variance and covariance were performed to identify group, time, and group $\times$ time interaction, as well as the effect of premeasures on any observed effects. Standardized changes in the mean of each measure were used to assess magnitudes of effects by dividing the changes by the appropriate between-athlete SDs in the pretest measures. Magnitudes of the standardized effects (effect size [ES]) were interpreted using thresholds of $0.2,0.6$, and 1.2 for small, moderate, and large, respectively. ${ }^{8}$ Standardized effects between -0.19 and 0.19 were termed trivial. The significance level was set at $P \leq .05$ for all analyses.

\section{Results}

Analysis of variance results revealed significant group and time effects for the injured, eyes-open condition (all $P \mathrm{~s} \leq .03$ ), but only time (pre/post) effects were observed in the AP sway data in the eyes-closed condition $(P=.03)$. Similarly, in the noninjured leg, group and time effects were apparent in the eyes-open condition, but no effects were observed in the eyes-closed condition. No interaction effects were detected.

When analyses of covariance were performed, prebalance variables were significantly related to pre versus post change scores in the injured (all $P \mathrm{~s} \leq .002$ ) and noninjured eyes-closed condition (all $P \mathrm{~s} \leq .001$ ), but not noninjured eyes-open condition (all $P \mathrm{~s}>.2$ ). Note that unadjusted means are presented in the figures below to give an unaltered representation of the changes observed.

\section{Single-Leg Balance Test}

Eyes Open-Total Sway. Only traditional rehabilitation significantly decreased eyes open-total sway in the injured leg (post-pre $\Delta:-169.8 ; P=.03 ; \mathrm{ES}=-0.54$; Figure $1 \mathrm{~A}$ and $1 \mathrm{~B}$ ). This decrease was not significantly greater than the decreases observed in the control group ( $-24.6 \mathrm{~mm} ; P=.07)$ or the UDP group $(-49.1 \mathrm{~mm}$; $P=.28)$. No significant pre to post changes or group differences were seen in eyes open-total sway for the uninjured leg.

Eyes Closed-Total Sway. Only unexpected disturbance training significantly decreased eyes closed-total sway in the injured leg (post-pre $\Delta:-271.8 \mathrm{~mm} ; P=.04 ; \mathrm{ES}=-0.61$ ) from pretest to posttest occasions; however, this decrease was not significantly greater than the decreases observed in the control group $(\Delta:-252.8 \mathrm{~mm} ; P=.79)$ or the traditional training group $(\Delta:-159.4 \mathrm{~mm} ; P=.59)$. The UDP group was the only group that improved eyes closed-total sway in the uninjured leg $(\Delta:-317.8 \mathrm{~mm} ; P=.02 ; \mathrm{ES}=-0.49)$, but there were no significant group differences.

Eyes Open-AP Sway. No significant decreases or group differences were observed in eyes open-AP sway in the injured leg. In the uninjured leg, eyes open-AP sway significantly decreased in the traditional training group only $(\Delta:-28.0 ; P=.04 ; \mathrm{ES}=-0.25)$.

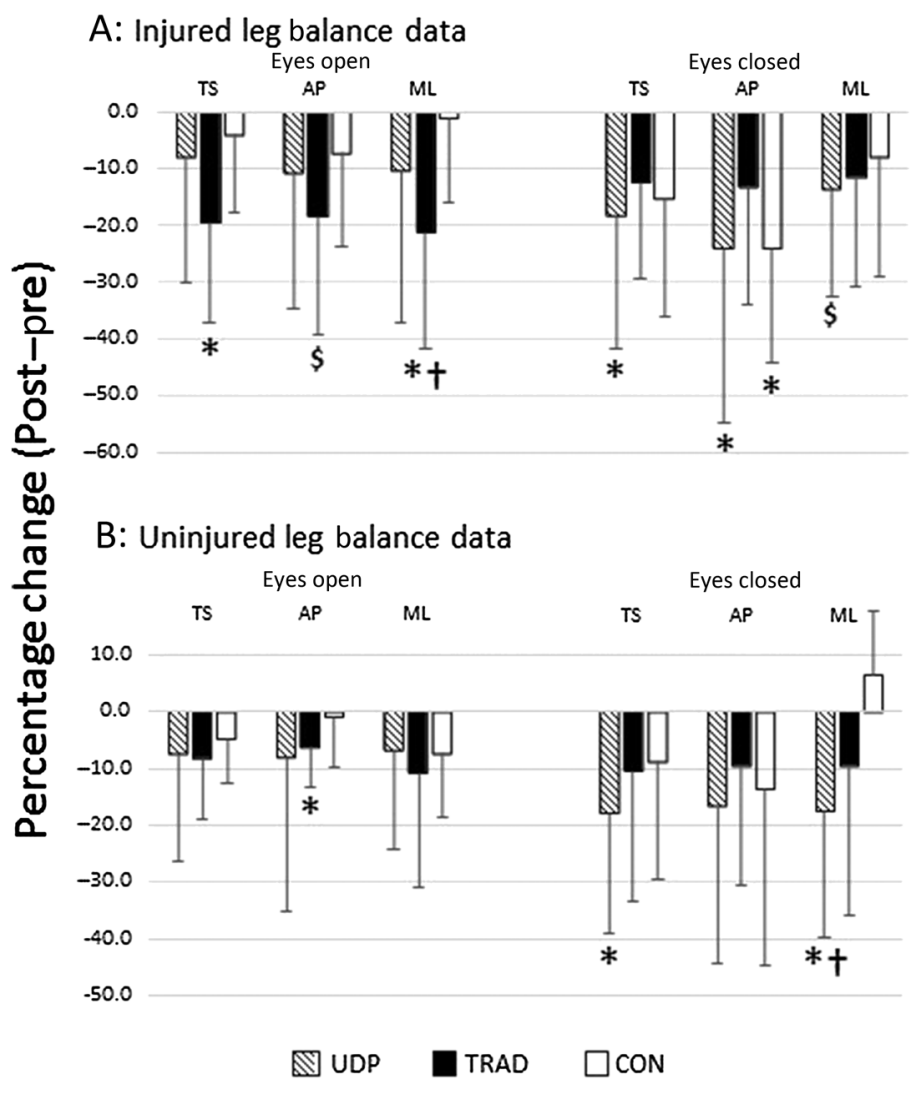

Figure 1 - Single leg balance eyes open and eyes closed data assessing percentage change in total sway (TS), anteroposterior (AP), and mediolateral (ML) movement. CON indicates nonexercise control group; TRAD, traditional rehabilitation program; UDP, unexpected disturbance program. *Significantly different to pretest. ${ }^{\dagger}$ Significantly different to control. ${ }^{\$} P$ value between .05 and .06 versus pretest.

This decrease was not significantly greater than the trivial change observed in the control group $(\Delta:-2.8 \mathrm{~mm} ; P=.19)$ or the decrease seen in the UDP group $(\Delta:-41.1 \mathrm{~mm} ; P=.88)$.

Eyes Closed-AP Sway. Both the control (post-pre $\Delta$ : $-268.5 \mathrm{~mm} ; P=.01 ; \mathrm{ES}=-0.65)$ and UDP $(\Delta:-211.2 \mathrm{~mm} ; P=$ .04 ; ES $=-0.76)$ groups decreased eyes closed-AP sway in the injured leg, with no group differences observed. No significant pre to post changes or group differences were observed in eyes closed-AP sway in the uninjured leg.

Eyes Open-ML Sway. Only traditional training significantly decreased eyes open-ML sway in the injured leg $(\Delta:-120.1 \mathrm{~mm}$; $P=.03 ; \mathrm{ES}=-0.53)$. This decrease was significantly greater than the trivial change observed in the control group $(\Delta:-0.1 \mathrm{~mm}$; $P=.03)$, but not the decrease in the UDP group $(\Delta:-42.8 \mathrm{~mm}$; $P=.38$ ). No significant pre to post changes or group differences were seen in eyes open-ML sway for the uninjured leg.

Eyes Closed-ML Sway. No significant pre to post changes or group differences were observed in eyes closed-ML sway in the injured leg, although the UDP group decreased by $134.3 \mathrm{~mm}$ $(P=.05 ; \mathrm{ES}=-0.50)$. A significant pre to post change was seen in eyes closed-ML sway in the uninjured leg of the UDP group $(\Delta:-205.9 \mathrm{~mm} ; P=.04 ; \mathrm{ES}=-0.47)$, with a significant difference between the UDP $(\Delta:-101.3 \mathrm{~mm})$ and control groups $(\Delta$ : $+66.2 \mathrm{~mm}), P=.01$. 


\section{Isometric Single-Leg Press Test}

Moderate increases in maximal isometric single-leg strength of the injured leg were seen in both the UDP $(\Delta: 26.2 \mathrm{~kg} ; P<.001$; $\mathrm{ES}=0.71)$ and traditional training groups $(\Delta: 20.0 \mathrm{~kg} ; P=.02$; $\mathrm{ES}=0.61$; Figure 2). After accounting for pretraining strength levels, the strength increase in the UDP group were significantly greater than the trivial change seen in the control group $(P=.003)$, but not the traditional training group $(P=.71)$. There was no significant difference between the control and traditional training groups $(P=.07)$.

In the uninjured leg, only the UDP group improved maximal isometric leg strength $(\Delta: 36.1 \mathrm{~kg} ; P<.001 ; \mathrm{ES}=1.08)$. After accounting for pretraining strength levels, this increase was significantly greater than the trivial change in strength in the control group $(1.0 \mathrm{~kg} ; P<.001)$ and the small increase seen in the traditional training group $(12.7 \mathrm{~kg} ; P=.04)$.

\section{Discussion}

The incorporation of an UDP in national-level athletes with an unilateral knee ligament injury produced similar, if not slightly inferior, improvements in balance with eyes open to a traditional rehabilitation program. An opposite trend, however, was observed in the eyes-closed condition, with slightly superior improvements with UDP compared with traditional training. We acknowledge that the lack of any observable interaction effect demonstrates that neither of the rehabilitation programs was consistently more effective than the control group. It is likely that a longer rehabilitation program (maybe 5-8 wk) would be needed to see differences between the rehabilitation programs. With respect to the unilateral strength adaptations, both the traditional and UDPs substantially improved strength in the injured leg, even after accounting for prestrength levels. Interestingly, the UDP also elicited a meaningful strength increase in the uninjured leg that was 23.4 and $35.1 \mathrm{~kg}$ greater than the increases seen in the traditional rehabilitation and control groups, respectively.

Balance proficiency is a primary motor skill associated with the integration of simple reflexes, proprioceptive inputs relayed to the cerebrum and cerebellum, and activation of the reticular formation, the vestibular apparatus, voluntary movements, and

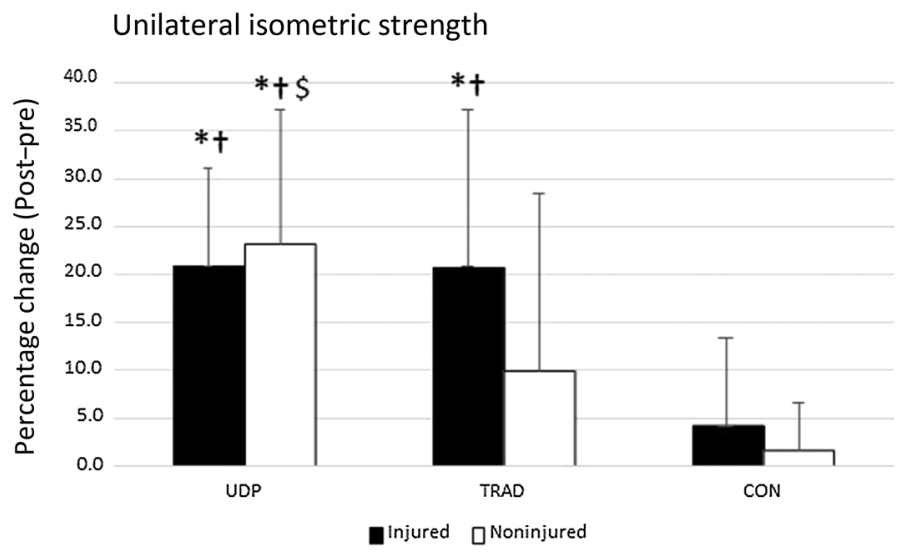

Figure 2 - Percentage change in single-leg isometric strength data. CON indicates nonexercise control group; TRAD traditional rehabilitation program; UDP, unexpected disturbance program. *Significantly different to pretest. ${ }^{\dagger}$ Significantly different to control. ${ }^{\$}$ Significantly different to TRAD. ocular information. ${ }^{9}$ Only the UDP group consistently improved all measures of balance (total sway distance, AP sway distance, and ML sway distance) in the injured leg compared with baseline testing when the eyes remained closed during the balance assessment. The magnitude of the improvements ranged from small to moderate (ES: -0.50 to -0.76 ) demonstrating that the improvements observed were meaningful. It is noteworthy that previous research has demonstrated rapid adaptations in brain regions associated with the integration of vestibular signals following sensorimotor training. ${ }^{10}$

An eyes-closed testing condition is commonly used in rehabilitation to assess neural plasticity in proprioceptive, vestibular, and even cerebellar disorders. ${ }^{11}$ As Demura et al ${ }^{12}$ demonstrated that central nervous disorders showed greater magnitude of ML sway compared with individuals with vestibular organ disorders, a decrease in sway in the eyes-closed conditions can potentially be interpreted as a neural adaptation. Given that the UDP increased reliance on nonvisual inputs, it is hypothesized that unexpected disturbance training improved proprioceptive, vestibular, and somatosensory feedback loops that transferred across to improvements in eyes-closed condition. Inglis et $\mathrm{al}^{13}$ have previously postulated an important role for somatosensory information from the lower limb in stimulating "centrally organized postural synergies." This adaptation is especially important, as it has been argued that vision provides a slow compensatory input to posture under normal conditions. ${ }^{14}$

While the UDP improved balance in the eyes-closed assessment, it was apparent that traditional rehabilitation practices were superior in enhancing eyes-open sway measures. It is clear that the visual system is of paramount importance for maintaining balance. ${ }^{9,15}$ Vision assists motor function by integrating spatial information enabling maintenance of balance via involuntary postural adjustments that can occur within $100 \mathrm{~ms} .{ }^{16}$ Given the positive, and potentially complementary, adaptations observed in the traditionally rehabilitated group when balance was assessed with eyes open, it can be recommended that this type of training be used in conjunction with unexpected disturbance training to further progress rehabilitative outcomes.

For the injured limb, there were significant improvements in unilateral maximal leg press strength in both UDP (ES: 0.71) and traditional rehabilitation groups (ES: 0.61) compared with the control group. These data coincide with Liu-Ambrose et al ${ }^{17}$ who showed that proprioceptive training can have a significant impact on neural activation in the early stages of strength gain following ACL reconstruction. Phase 3 of rehabilitation can be considered to bear similarities to these early stages, as ACL injuries result in loss of strength in quadriceps and hamstring ${ }^{18}$ that have been related to the inhibition of afferent reaction from the ACL to gamma motor neurons. ${ }^{19}$

Interestingly, UDP also elicited significantly superior gains in strength in the uninjured leg when adjusting for baseline strength characteristics. Previous work has shown perturbation training to be effective in improving explosive strength in uninjured elite athletes, ${ }^{1}$ neural activation, ${ }^{20,21}$ and muscular coordination. ${ }^{22}$ These adaptations may explain the gains seen in the contralateral limb as well as potentially underlie the improvements observed in balance metrics in the uninjured leg following the UDP (or these improvements may simply reflect the increase in lower-limb strength). ${ }^{23}$ Such adaptations are especially important given the known neuromuscular asymmetries associated with ACL deficiency. ${ }^{24}$

Another important aspect of injury rehabilitation is overcoming the fear of movements that could cause reinjury, known as 
kinesiophobia. Kinesiophobia has been closely correlated with the lack of or failure to return to sport, ${ }^{25,26}$ even in the absence of knee pain. ${ }^{27}$ In the current investigation, the unexpected perturbations challenged the sensorimotor functions of the injured athletes. We believe that these interventions could assist with overcoming kinesiophobia and fear of reinjury given previous research with elite athletes. ${ }^{2}$

\section{Conclusions}

The purpose of rehabilitation (particularly postsurgical rehabilitation) is to return injured athletes to their initial functional capacity and playing level before injury in an optimal time span, as well as to reduce the risk of reinjury upon return to play. Unfortunately, given the high reinjury rates, it is clear that return-to-play protocols after knee surgery are suboptimal. We acknowledge that the small sample size, short-time frame, and possibility for external confounders influence the individual testing and training sessions; however, the high level of ecological validity suggests that UDP could potentially improve neural adaptations to shorten phase 3 of the rehabilitation by improving strength and proprioception. Given the complementary adaptations shown here, we conclude that an UDP may provide an effective adjunct to traditional rehabilitation protocols and improve return-to-play outcomes.

\section{References}

1. Teichmann J, Suwarganda EK, Beaven CM, et al. A three week unexpected disturbance program improves physical performance of elite female athletes. J Sport Rehabil. 2016;27(1):1-7.

2. Teichmann J, Suwarganda EK, Lendewig C, et al. Unexpecteddisturbance program for rehabilitation of high-performance athletes. J Sport Rehabil. 2016;25(2):126-132. PubMed ID: 25658597 doi:10. 1123/jsr.2014-0280

3. Hertel J, Denegar CR. A rehabilitation paradigm for restoring neuromuscular control following athletic injury. Athl Ther Today. 1998; 3(5):12-16. doi:10.1123/att.3.5.12

4. Barber-Westin SD, Noyes FR. Factors used to determine return to unrestricted sports activities after anterior cruciate ligament reconstruction. Arthroscopy. 2011;27(12):1697-1705. PubMed ID: 22137326 doi:10.1016/j.arthro.2011.09.009

5. Lentz TA, Zeppieri G Jr, Tillman SM, et al. Return to preinjury sports participation following anterior cruciate ligament reconstruction: contributions of demographic, knee impairment, and self-report measures. J Orthop Sports Phys Ther. 2012;42(11):893-901. PubMed ID: 22951437 doi:10.2519/jospt.2012.4077

6. Hägglund M, Waldén M, Ekstrand J. Previous injury as a risk factor for injury in elite football: a prospective study over two consecutive seasons. Br J Sports Med. 2006;40(9):767-772. doi:10.1136/bjsm. 2006.026609

7. Taube W, Schubert M, Gruber M, Beck S, Faist M, Gollhofer A. Direct corticospinal pathways contribute to neuromuscular control of perturbed stance. J Appl Physiol. 2006;101(2):420-429. PubMed ID: 16601305 doi:10.1152/japplphysiol.01447.2005

8. Hopkins WG, Marshall SW, Batterham AM, Hanin J. Progressive statistics for studies in sports medicine and exercise science. Med Sci Sports Exerc. 2009;41(1):3-13. PubMed ID: 19092709 doi:10.1249/ MSS.0b013e31818cb278

9. Ribadi H, Rider RA, Toole T. A comparison of static and dynamic balance in congenitally blind, sighted, and sighted blindfolded adolescents. Adapt Phys Activ Q. 1987;4(3):220-225. doi:10.1123/ apaq.4.3.220

10. Taubert M, Draganski B, Anwander A, et al. Dynamic properties of human brain structure: learning-related changes in cortical areas and associated fiber connections. J Neurosci. 2010;30(35):11670-11677. PubMed ID: 20810887 doi:10.1523/JNEUROSCI.2567-10.2010

11. Yelnik AP, Tasseel Ponche S, Andriantsifanetra C, Provost C, Calvalido A, Rougier P. Walking with eyes closed is easier than walking with eyes open without visual cues: the Romberg task versus the goggle task. Ann Phys Rehabil Med. 2015;58(6):332-335. PubMed ID: 26439522 doi:10.1016/j.rehab.2015.08.004

12. Demura S, Kitabayashi T, Kimura A, Matsuzawa J. Body sway characteristics during static upright posture in healthy and disordered elderly. J Physiol Anthropol Appl Human Sci. 2005;24(5):551-555. PubMed ID: 16237264 doi:10.2114/jpa.24.551

13. Inglis JT, Horak FB, Shupert CL, Jones-Rycewicz C. The importance of somatosensory information in triggering and scaling automatic postural responses in humans. Exp Brain Res. 1994;101(1):159-164. PubMed ID: 7843295 doi:10.1007/BF00243226

14. Lestienne F, Soechting J, Berthoz A. Postural readjustments induced by linear motion of visual scenes. Exp Brain Res. 1977;28(3-4): 363-384. PubMed ID: 885185

15. Travis RC. An experimental analysis of dynamic and static equilibrium. J Exp Psychol. 1945;35(3):216-234. doi:10.1037/ h0059788

16. Nashner L, Berthoz A. Visual contribution to rapid motor responses during postural control. Brain Res. 1978;150(2):403-407. PubMed ID: 678978 doi:10.1016/0006-8993(78)90291-3

17. Liu-Ambrose T, Taunton JE, MacIntyre D, McConkey P, Khan KM. The effects of proprioceptive or strength training on the neuromuscular function of the ACL reconstructed knee: a randomized clinical trial. Scand J Med Sci Sports. 2003;13(2):115-123. PubMed ID: 12641643 doi:10.1034/j.1600-0838.2003.02113.x

18. Konishi Y, Fukubayashi T, Takeshita D. Mechanism of quadriceps femoris muscle weakness in patients with anterior cruciate ligament reconstruction. Scand J Med Sci Sports. 2002;12(6):371-375. PubMed ID: 12453165 doi:10.1034/j.1600-0838.2002.01293.x

19. Papadonikolakis A, Cooper L, Stergiou N, Georgoulis AD, Soucacos PN. Compensatory mechanisms in anterior cruciate ligament deficiency. Knee Surg Sports Traumatol Arthrosc. 2003;11(4):235-243. PubMed ID: 12700888 doi:10.1007/s00167-003-0367-6

20. Gruber M, Gollhofer A. Impact of sensorimotor training on the rate of force development and neural activation. Eur J Appl Physiol. 2004;92(1-2):98-105. PubMed ID: 15024669 doi:10.1007/s00421004-1080-y

21. Lauber B, Keller M, Gollhofer A, Müller E, Taube W. Spinal reflex plasticity in response to alpine skiing in the elderly. Scand J Med Sci Sports. 2011;21(suppl 1):62-68. doi:10.1111/j.1600-0838.2011. 01343.x

22. Chmielewski TL, Hurd WJ, Rudolph KS, Axe MJ, Snyder-Mackler L. Perturbation training improves knee kinematics and reduces muscle co-contraction after complete unilateral anterior cruciate ligament rupture. Phys Ther. 2005;85(8):740-749. PubMed ID: 16048422 doi:10.1093/ptj/85.8.740

23. Lin SI, Woollacott M. Association between sensorimotor function and functional and reactive balance control in the elderly. Age Ageing. 2005;34(4):358-363. PubMed ID: 15899911 doi:10.1093/ ageing/afi089

24. Gardinier ES, Manal K, Buchanan TS, Snyder-Mackler L. Gait and neuromuscular asymmetries after acute anterior cruciate ligament rupture. Med Sci Sports Exerc. 2012;44(8):1490-1496. PubMed ID: 22330021 doi:10.1249/MSS.0b013e31824d2783 
25. Ardern CL, Webster KE, Taylor NF, Feller JA. Return to sport following anterior cruciate ligament reconstruction surgery: a systematic review and meta-analysis of the state of play. Br J Sports Med. 2011;45(7):596-606. PubMed ID: 21398310 doi:10.1136/bjsm. 2010.076364

26. Paterno MV, Flynn K, Thomas S, Schmitt LC. Self-reported fear predicts functional performance and second ACL injury after ACL reconstruction and return to sport: a pilot study. Sports Health.
2018;10(3):228-233. PubMed ID: 29272209 doi:10.1177/ 1941738117745806

27. Flanigan DC, Everhart JS, Pedroza A, Smith T, Kaeding CC. Fear of reinjury (kinesiophobia) and persistent knee symptoms are common factors for lack of return to sport after anterior cruciate ligament reconstruction. Arthroscopy. 2013;29(8):1322-1329. PubMed ID: 23906272 doi:10.1016/j.arthro.2013.05.015 